\title{
THE UNIQUENESS THEOREMS OF MEROMORPHIC FUNCTIONS SHARING THREE VALUES AND ONE PAIR OF POLYNOMIALS
}

\author{
XiaO-Min Li And Hong-Xun Yi
}

\begin{abstract}
In this paper, we deal with a uniqueness theorem of two nonconstant meromorphic functions that share three values and one pair of polynomials. The results in this paper improve those given by G. G. Gundersen, G. Brosch and other authors.
\end{abstract}

\section{Introduction and main results}

In this paper, by meromorphic functions we will always mean meromorphic functions in the complex plane. We adopt the standard notations in the Nevanlinna theory of meromorphic functions as explained in [8] and [18]. It will be convenient to let $E$ denote any set of positive real numbers of finite linear measure, not necessarily the same at each occurrence. For a nonconstant meromorphic function $h$, we denote by $T(r, h)$ the Nevanlinna characteristic of $h$ and by $S(r, h)$ any quantity satisfying $S(r, h)=o\{T(r, h)\}(r \rightarrow \infty, r \notin E)$.

Let $f$ and $g$ be two nonconstant meromorphic functions, and let $a$ be a value in the extended plane. We say that $f$ and $g$ share the value $a \mathrm{CM}$, provided that $f$ and $g$ have the same $a$-points with the same multiplicities. Similarly, we say that $f$ and $g$ share the value $a \mathrm{IM}$, provided that $f$ and $g$ have the same $a$-points ignoring multiplicities (see [18]). We say that $a$ is a small function of $f$, if $a$ is a meromorphic function satisfying $T(r, a)=S(r, f)$ as $r \rightarrow \infty$. In addition, we need the following definition.

Definition 1.1 (see [3, Definition 1]). Let $p$ be a positive integer and $a \in$ $C \cup\{\infty\}$. Then by $N_{p)}(r, 1 /(f-a))$ we denote the counting function of those zeros of $f-a$ (counted with proper multiplicities) whose multiplicities are not greater than $p$, by $\bar{N}_{p)}(r, 1 /(f-a))$ we denote the corresponding reduced

Received February 11, 2009.

2000 Mathematics Subject Classification. Primary 30D30, 30 D35.

Key words and phrases. meromorphic functions, weighted sharing values, uniqueness theorems.

Project supported by the NSFC (No. 10771121), the NSF of Shandong Province, China (No. Z2008A01), the NSFC \& RFBR (Joint Project) (No. 10911120056), and the NSF of Shandong Province, China (No.ZR2009AM008). 
counting function (ignoring multiplicities). By $N_{(p}(r, 1 /(f-a))$ we denote the counting function of those zeros of $f-a$ (counted with proper multiplicities) whose multiplicities are not less than $p$, by $\bar{N}_{(p}(r, 1 /(f-a))$ we denote the corresponding reduced counting function (ignoring multiplicities).

Let $f$ and $g$ be two nonconstant meromorphic functions, and let $a$ be a value in the extended plane. Let $S$ be a subset of distinct elements in the extended plane. Next we define

$$
E_{f}(S)=\bigcup_{a \in S}\{z: f(z)=a\},
$$

where each $a$-point of $f$ with multiplicity $m$ is repeated $m$ times in $E_{f}(S)$ (see [6]). Similarly, we define

$$
\bar{E}_{f}(S)=\bigcup_{a \in S}\{z: f(z)=a\},
$$

where each point in $\bar{E}_{f}(\{a\})$ is counted only once. We say that $f$ and $g$ share the set $S \mathrm{CM}$, provided $E_{f}(S)=E_{g}(S)$. We say that $f$ and $g$ share the set $S$ IM, provided $\bar{E}_{f}(S)=\bar{E}_{g}(S)$. Next by the notation $f=a \Longrightarrow g=a$ we denote $\bar{E}_{f}(\{a\}) \subseteq \bar{E}_{g}(\{a\})$

In 1926, R. Nevanlinna proved the following theorem.

Theorem A (see [17]). If $f$ and $g$ are nonconstant meromorphic functions that share five values $I M$, then $f=g$.

Theorem B (see [17]). If $f$ and $g$ are distinct nonconstant meromorphic functions that share four values $a_{1}, a_{2}, a_{3}$ and $a_{4} C M$, then $f$ is a Möbius transformation of $g$, two of the shared values, say $a_{1}$ and $a_{2}$, are Picard values, and the cross ratio $\left(a_{1}, a_{2}, a_{3}, a_{4}\right)=-1$.

In 1979, G. G. Gundersen proved the following theorem, which improved Theorem B.

Theorem C (see [7, Theorem 1]). Let $f$ and $g$ be two distinct nonconstant meromorphic functions such that $f$ and $g$ share three values $C M$ and share a fourth value IM. Then $f$ and $g$ share all four values $C M$, and hence the conclusion of Theorem B holds.

In 1989, G. Brosch proved the following theorem, which improved Theorem $\mathrm{B}$ and Theorem $\mathrm{C}$.

Theorem D (see [5]). Let $f$ and $g$ be two distinct nonconstant meromorphic functions such that $f$ and $g$ share 0,1 and $\infty C M$, and let $a$ and $b$ be two distinct complex numbers such that $a, b \notin\{0,1\}$. If $f-a$ and $g-b$ share 0 IM, then $f$ is a Möbius transformation of $g$.

Regarding Theorem D, it is natural to ask the following two questions. 
Question 1.1 (see [9]). Is it really possible to relax in any way the nature of sharing any one of 0,1 and $\infty$ in Theorem $\mathrm{D}$ ?

Question 1.2. What can be said if $a$ and $b$ in Theorem D are replaced with two distinct nonconstant polynomials $P_{1}$ and $P_{2}$ respectively?

Recently many mathematicians in the world have done a lot of research works concerning Question 1.1, such as T. C. Alzahary [2, 3], I. Lahiri and P. Sahoo [11], X. M. Li and H. X. Yi [12], etc. In these research works, the notion of weighted sharing of values has been used, which measures how close a shared value is to being shared IM or to being shared CM. The notion is explained in the following definition.

Definition 1.2 (see [10, Definition 4]). Let $k$ be a nonnegative integer or infinity. For any $a \in C \cup\{\infty\}$, we denote by $E_{k}(a, f)$ the set of all $a$-points of $f$, where an $a$-point of multiplicity $m$ is counted $m$ times if $m \leq k$, and $k+1$ times if $m>k$. If $E_{k}(a, f)=E_{k}(a, g)$, we say that $f, g$ share the value $a$ with weight $k$.

Remark 1.1. Definition 1.2 implies that if $f, g$ share a value $a$ with weight $k$, then $z_{0}$ is a zero of $f-a$ with multiplicity $m(\leq k)$ if and only if it is a zero of $g-a$ with multiplicity $m(\leq k)$, and $z_{0}$ is a zero of $f-a$ with multiplicity $m(>k)$, if and only if it is a zero of $g-a$ with multiplicity $n(>k)$, where $m$ is not necessarily equal to $n$. Throughout this paper, we write $f, g$ share $(a, k)$ to mean that $f, g$ share the value $a$ with weight $k$. Clearly, if $f, g$ share $(a, k)$, then $f, g$ share $(a, p)$ for all integer $p, 0 \leq p<k$. Also we note that $f, g$ share a value $a$ IM or CM if and only if $f, g$ share $(a, 0)$ or $(a, \infty)$, respectively.

In this paper, we will prove the following two theorems that deals with Question 1.2.

Theorem 1.1. Let $f$ and $g$ be two distinct nonconstant meromorphic functions such that $f$ and $g$ share $0,1, \infty C M$, and let $P_{1}$ and $P_{2}$ be two nonconstant polynomials such that $P_{1} \not \equiv P_{2}$. If $f-P_{1}$ and $g-P_{2}$ share $0 I M$, then $f$ and $g$ are transcendental meromorphic functions and satisfy one of the following three relations: (i) $f+g=1$ with $P_{1}+P_{2}=1$; (ii) $f=\frac{P_{1}}{P_{2}} \cdot g$; (iii) $f=\frac{P_{1}-1}{P_{2}-1} \cdot g+\frac{P_{2}-P_{1}}{P_{2}-1}$.

Theorem 1.2. Let $f$ and $g$ be two nonconstant entire functions that share 0 and $1 C M$, and let $P_{1}$ and $P_{2}$ be two nonconstant polynomials such that $P_{1} \not \equiv P_{2}$. If $f-P_{1}=0 \Longrightarrow g-P_{2}=0$, then $f=g$.

\section{Some lemmas}

Lemma 2.1 (see [7, Theorem 3]). Let $f$ and $g$ share $0,1, \infty$ IM. Then

$$
\left(\frac{1}{3}+o(1)\right) T(r, g)<T(r, f)<(3+o(1)) T(r, g)(r \notin E) .
$$


Lemma 2.2 (see [19, Lemma 2.6]). Let $f$ and $g$ be two distinct nonconstant meromorphic functions that share $\left(0, k_{1}\right),\left(1, k_{2}\right)$ and $\left(\infty, k_{3}\right)$, where $k_{1}, k_{2}$ and $k_{3}$ are three positive integers satisfying

$$
k_{1}+k_{2}+k_{3}>k_{1} k_{2} k_{3}+2 .
$$

Then

$$
\begin{array}{ll}
\text { (i) } & \bar{N}_{(2}\left(r, \frac{1}{f}\right)+\bar{N}_{(2}\left(r, \frac{1}{f-1}\right)+\bar{N}_{(2}(r, f)=S(r, f) ; \\
\text { (ii) } & \bar{N}_{(2}\left(r, \frac{1}{g}\right)+\bar{N}_{(2}\left(r, \frac{1}{g-1}\right)+\bar{N}_{(2}(r, g)=S(r, f) .
\end{array}
$$

Lemma 2.3. Let $f$ and $g$ be two nonconstant rational functions that share $\left(0, k_{1}\right),\left(1, k_{2}\right)$ and $\left(\infty, k_{3}\right)$, where $k_{1}, k_{2}, k_{3}$ are three positive integers satisfying (2.1). Then $f=g$.

Proof. Suppose that $f \not \equiv g$. From the fact that $f$ and $g$ are two nonconstant rational functions we have

$$
T(r, f) \leq A_{1} \log r, \quad T(r, g) \leq A_{2} \log r,
$$

where $A_{1}$ and $A_{2}$ are positive numbers. Let

$$
\alpha_{1}=\frac{f^{\prime}}{f-1}-\frac{g^{\prime}}{g-1}
$$

and

$$
\beta_{1}=\frac{f^{\prime}}{f}-\frac{g^{\prime}}{g} .
$$

From (2.3), (2.4) and Lemma 2.2 we get

$$
T\left(r, \alpha_{1}\right)+T\left(r, \beta_{1}\right)=S(r, f),
$$

which together with $\lim _{\substack{r \rightarrow \infty \\ r \notin E}} S(r, f) / T(r, f)=0$ implies

$$
\lim _{\substack{r \rightarrow \infty \\ r \notin E}} \frac{T\left(r, \alpha_{1}\right)+T\left(r, \beta_{1}\right)}{T(r, f)}=0 .
$$

From the fact that $f$ and $g$ are two nonconstant rational functions we see that $\alpha_{1}$ and $\beta_{1}$ are rational functions. Thus

$$
T\left(r, \alpha_{1}\right)+T\left(r, \beta_{1}\right)=O(\log r) .
$$

Suppose that one of $\alpha_{1}$ and $\beta_{1}$ is not a constant. Then there exists some positive number $A_{3}$ such that

$$
T\left(r, \alpha_{1}\right)+T\left(r, \beta_{1}\right) \geq A_{3} \log r .
$$

From the left inequality of (2.2) and (2.8) we get

$$
\frac{T\left(r, \alpha_{1}\right)+T\left(r, \beta_{1}\right)}{T(r, f)} \geq \frac{A_{3}}{A_{1}},
$$


which contradicts (2.6). Thus $\alpha_{1}$ and $\beta_{1}$ are constants, say $\alpha_{1}=c_{1}$ and $\beta_{1}=c_{2}$. Then (2.3) and (2.4) can be rewritten as

$$
\frac{f^{\prime}}{f-1}-\frac{g^{\prime}}{g-1}=c_{1}
$$

and

$$
\frac{f^{\prime}}{f}-\frac{g^{\prime}}{g}=c_{2}
$$

respectively. From (2.9) and (2.10) we get

$$
f-1=A_{4}(g-1)
$$

and

$$
f=A_{5} g,
$$

where $A_{4}(\neq 0)$ and $A_{5}(\neq 0)$ are two complex numbers. From (2.11) and (2.12) we get $A_{5} g-1=A_{4}(g-1)$, which implies $A_{4}=A_{5}$, and so it follows from (2.11) and (2.12) that $A_{4}=A_{5}=1$. Thus $f=g$, which contradicts the above supposition. Lemma 2.3 is thus completely proved.

Lemma 2.4 (see [18, Theorem 1.5]). If $f$ is a transcendental meromorphic function in the complex plane, then $\lim _{r \rightarrow \infty} T(r, f) / \log r=\infty$.

Let $f$ and $g$ be two nonconstant meromorphic functions in the complex plane, and $a$ be a value in the extended plane. Let $\bar{N}_{E}(r, a)$ "count" those points in $\bar{N}(r, 1 /(f-a))$, where $a$ is taken by $f$ and $g$ with the same multiplicity, and each point is counted only once, and let $\bar{N}_{0}(r, a)$ be the reduced counting function of the common $a$-points of $f$ and $g$ in $\bar{N}(r, 1 /(f-a))$, where $\bar{N}(r, 1 /(f-\infty))$ means $\bar{N}(r, f)$. We say that $f$ and $g$ share the value $a \mathrm{CM}^{*}$, if

$$
\bar{N}\left(r, \frac{1}{f-a}\right)-\bar{N}_{E}(r, a)=S(r, f)
$$

and

$$
\bar{N}\left(r, \frac{1}{g-a}\right)-\bar{N}_{E}(r, a)=S(r, g) .
$$

We say that $f$ and $g$ share the value $a \mathrm{IM}^{*}$, if

$$
\bar{N}\left(r, \frac{1}{f-a}\right)-\bar{N}_{0}(r, a)=S(r, f)
$$

and

$$
\bar{N}\left(r, \frac{1}{g-a}\right)-\bar{N}_{0}(r, a)=S(r, g) .
$$

The two notions can be found in [13] or [18]. If there exist four small functions $\alpha_{1}, \alpha_{2}, \alpha_{3}, \alpha_{4}$ of $f$ and $g$ such that $f=\left(\alpha_{1} g+\alpha_{2}\right) /\left(\alpha_{3} g+\alpha_{4}\right)$, where $\alpha_{1} \alpha_{4}-$ $\alpha_{2} \alpha_{3} \not \equiv 0$, then we say that $f$ is a quasi-Möbius transformation of $g$ (see [13] or [18]). 
Lemma 2.5 (see [13, Theorem 3]). Let $f$ and $g$ be two distinct nonconstant meromorphic functions that share $0,1, \infty C M^{*}$, and let $a \neq 0,1, \infty$ be a small function of $f$ and $g$. If $T(r, f) \neq N(r, 1 /(f-a))+S(r, f)$, then $f$ is a quasiMöbius transformation of $g$ such that $f$ and $g$ satisfy one of the following three relations: (i) $f=a g$; (ii) $f+(a-1) g=a$; $\quad$ (iii) $(f-a)(g+a-1)=a(1-a)$.

Lemma 2.6 (see [1, Lemma 3]). Let $f$ and $g$ be two distinct nonconstant meromorphic functions that share $0,1, \infty$ CM. If $f$ is a Möbius transformation of $g$, then $f$ and $g$ satisfy one of the following six relations.
(i) $f g=1$;
(ii) $(f-1)(g-1)=1$;
(iii) $f+g=1$;
(iv) $f=c g$;
(v) $f-1=c(g-1)$;
(vi) $\{(c-1) f+1\} \cdot\{(c-1) g-c\}=-c$;

where $c \neq 0,1$ is a complex number.

Lemma 2.7 (see [18, Theorem 1.62]). Let $f_{1}, f_{2}, \ldots, f_{n}$ be non-constant meromorphic functions, and let $f_{n+1}(\not \equiv 0)$ be a meromorphic function such that $\sum_{j=1}^{n+1} f_{j}=1$. If there exists a subset $I \subseteq R^{+}$satisfying mes $I=\infty$ such that

$\sum_{i=1}^{n+1} N\left(r, \frac{1}{f_{i}}\right)+n \sum_{\substack{i=1 \\ i \neq j}}^{n+1} \bar{N}\left(r, f_{i}\right)<(\lambda+o(1)) T\left(r, f_{j}\right)(r \rightarrow \infty, r \in I, j=1,2, \ldots, n)$,

where $\lambda<1$. Then $f_{n+1}=1$.

Lemma 2.8. Let $f$ and $g$ be two transcendental meromorphic functions that share $0,1, \infty C M$, and let $P$ be a nonconstant polynomial. If

$$
T(r, f) \neq N\left(r, \frac{1}{f-P}\right)+S(r, f)
$$

then $f=g$.

Proof. Let $f$ and $g$ be distinct. First, from (2.13) and Lemma 2.5 we see that $f$ and $g$ satisfy one of the three relations: $f=P g, f+(P-1) g=P$ and $(f-P)(g+P-1)=P(1-P)$. By the condition that $f$ and $g$ share $0,1, \infty$ $\mathrm{CM}$ we have

$$
\frac{f-1}{g-1}=e^{\alpha}
$$

and

$$
\frac{f}{g}=e^{\alpha-\beta},
$$

where $\alpha$ and $\beta$ are entire functions. From the supposition $f \not \equiv g$ we have $e^{\alpha} \not \equiv 1, e^{\beta} \not \equiv 1$ and $e^{\alpha-\beta} \not \equiv 1$. Combining (2.14) and (2.15), we get

$$
f=\frac{e^{\alpha}-1}{e^{\beta}-1}
$$


and

$$
g=\frac{e^{-\alpha}-1}{e^{-\beta}-1}
$$

If one of $e^{\alpha}, e^{\beta}$ and $e^{\beta-\alpha}$ is a constant, from (2.14) and (2.15) we see that $f$ is a Möbius transformation of $g$. Thus $f$ and $g$ satisfy one of the six relations (i)-(vi) of Lemma 2.6. From this we see that there exist two distinct Picard exceptional values of $f$ and $g$. This together with Nevanlinna's three small functions theorem (see [18, Theorem 1.36]) implies

$$
T(r, f)=N\left(r, \frac{1}{f-P}\right)+S(r, f),
$$

which contradicts (2.13). Thus none of $e^{\alpha}, e^{\beta}$ and $e^{\beta-\alpha}$ is a constant. If $f$ and $g$ satisfy $f=P g$, from $(2.15)$ we have $P e^{\beta-\alpha}=1$, which is impossible. If $f$ and $g$ satisfy

$$
f+(P-1) g=P,
$$

by substituting (2.16) and (2.17) into (2.19) we get

$$
e^{\alpha}-e^{\beta}+(1-P) \cdot e^{\beta-\alpha}=1-P .
$$

Since none of $e^{\alpha}, e^{\beta}$ and $e^{\beta-\alpha}$ is a constant, from (2.20) and Lemma 2.7 we get a contradiction. If $f$ and $g$ satisfy

$$
(f-P)(g+P-1)=P(1-P)
$$

by substituting (2.16) and (2.17) into (2.21) we get

$$
P e^{\alpha+\beta}-e^{\beta}+(1-P) e^{\alpha}-P e^{2 \beta}+P e^{2 \beta-\alpha}+(1-P) e^{\beta-\alpha}=1-P .
$$

If $e^{\alpha+\beta}$ is a constant, then $e^{2 \beta-\alpha}$ is not a constant. This together with (2.22) and Lemma 2.7 implies $P e^{\alpha+\beta}=1-P$, which is impossible. If $e^{2 \beta-\alpha}$ is a constant, then $e^{\alpha+\beta}$ is not a constant. This together with (2.22) and Lemma 2.7 implies $e^{2 \beta-\alpha}=1-P$, which is impossible.

If $e^{\alpha+\beta}$ and $e^{2 \beta-\alpha}$ are not constants, from (2.22) and Lemma 2.7 we also get a contradiction. Lemma 2.8 is thus completely proved.

Lemma 2.9 (see [14, Theorem 4.1]). Let $f$ and $g$ be two distinct nonconstant meromorphic functions such that $f$ and $g$ share $\left(0, k_{1}\right),\left(1, k_{2}\right)$ and $\left(\infty, k_{3}\right)$, where $k_{1}, k_{2}$ and $k_{3}$ are three positive integers satisfying (2.1), and let a $\not \equiv$ $0,1, \infty$ be a nonconstant small meromorphic function of $f$ and $g$. Then either $N_{(3}(r, 1 /(f-a))+N_{(3}(r, 1 /(g-a))=S(r, f)$ holds, or $f$ and $g$ satisfy one of the three relations (i)-(iii) of Lemma 2.5.

Lemma 2.10 (see [13, Theorem 2]). Let $f$ and $g$ be two distinct nonconstant meromorphic functions that share $0,1, \infty C M^{*}$, and let $a(\not \equiv 0,1, \infty)$ and $b(\not \equiv 0,1, \infty)$ be two small functions of $f$ and $g$ such that $a \not \equiv b$. If $f-a$ and $g-b$ share $0 C M^{*}$, then $f$ is a quasi-Möbius transformation of $g$. 
Lemma 2.11 (see [15, Lemma 2.6]). Let $f$ and $g$ be two distinct nonconstant meromorphic functions such that $f$ and $g$ share $0,1, \infty$ IM. If $f$ is a quasiMöbius transformation of $g$, then $f$ and $g$ assume one of the following six relations.

(i) $f \cdot g=1$;

(ii) $\quad(f-1)(g-1)=1$;

(iii) $f+g=1$;

(iv) $f=c g$;

(v) $f-1=c(g-1)$

(vi) $[(c-1) f+1] \cdot[(c-1) g-c]=-c$,

where $c \not \equiv 0,1, \infty$ is a small function of $f$ and $g$.

Lemma 2.12 (see [18, Theorem 2.14]). Let $P_{1}$ and $P_{2}$ be two nonconstant polynomials, and let $a$ and $b$ be two distinct finite complex numbers. If $P_{1}$ and $P_{2}$ share $a$ and $b I M$, then $P_{1}=P_{2}$.

Lemma 2.13 (see [20, Lemma 1]). Let $h$ be a nonconstant entire function. Then $T\left(r, h^{\prime}\right)=o\left(T\left(r, e^{h}\right)\right)(r \rightarrow \infty, r \notin E)$.

Lemma 2.14 (see [22, Lemma 6]). Let $f_{1}$ and $f_{2}$ be two nonconstant meromorphic functions satisfying $\bar{N}\left(r, f_{j}\right)+\bar{N}\left(r, 1 / f_{j}\right)=S(r)(j=1,2)$. Then either $\bar{N}_{0}\left(r, 1 ; f_{1}, f_{2}\right)=S(r)$ or there exist two integers $s, t(|s|+|t|>0)$ such that $f_{1}^{s} f_{1}^{t} \equiv 1$, where and in what follows, $\bar{N}_{0}\left(r, 1 ; f_{1}, f_{2}\right)$ denotes the reduced counting function of $f_{1}$ and $f_{2}$ related to the common 1-points, and $T(r)=T\left(r, f_{1}\right)+T\left(r, f_{2}\right), S(r)=o(T(r))(r \rightarrow \infty, r \notin E)$ only depending on $f_{1}$ and $f_{2}$.

Lemma 2.15 (see [16]). Let $f$ be a nonconstant meromorphic function, and let $F=\sum_{k=0}^{p} a_{k} f^{k} / \sum_{j=0}^{q} b_{j} f^{j}$ be an irreducible rational function in $f$, where the coefficients $\left\{a_{k}\right\}$ and $\left\{b_{j}\right\}$ are small functions of $f$, and $a_{p} \not \equiv 0, b_{q} \not \equiv 0$. Then $T(r, F)=d T(r, f)+S(r, f)$, where $d=\max \{p, q\}$.

Lemma 2.16 (see [21, Corollary 2.2]). Let $f$ and $g$ be two distinct nonconstant entire functions that share two distinct values CM. Then

$$
\lim _{\substack{r \rightarrow \infty \\ r \notin E}} T(r, f) / T(r, g)=1 .
$$

\section{Proof of theorems}

Proof of Theorem 1.1. Suppose that $f \not \equiv g$. From Lemma 2.1 and the condition that $f$ and $g$ share $0,1, \infty \mathrm{CM}$ we see that $f$ is a rational function if and only if $g$ is a rational function, and $f$ is a transcendental meromorphic function if and only if $g$ is a transcendental meromorphic function. Suppose that $f$ and $g$ are two rational functions. Then it follows from Lemma 2.3 that $f=$ $g$, which contradicts above supposition. Next we suppose that $f$ and $g$ are two transcendental meromorphic functions. Then from Lemma 2.4 we have $T\left(r, P_{1}\right)+T\left(r, P_{2}\right)=o(T(r, f))$, and so $P_{1}, P_{2}$ are small functions of $f$ and 
$g$. From $f \not \equiv g$ and the assumptions of Theorem 1.1 we have (2.14)-(2.17). Suppose that

$$
T(r, f) \neq N\left(r, \frac{1}{f-P_{1}}\right)+S(r, f) .
$$

Then from (3.1) and Lemma 2.8 we get $f=g$, which contradicts the above supposition. Similarly, if

$$
T(r, g) \neq N\left(r, \frac{1}{g-P_{2}}\right)+S(r, f),
$$

then $f=g$, which contradicts the above supposition. Thus

$$
T(r, f)=N\left(r, \frac{1}{f-P_{1}}\right)+S(r, f)
$$

and

$$
T(r, g)=N\left(r, \frac{1}{g-P_{2}}\right)+S(r, f) .
$$

If one of $f=P_{1} g, f+\left(P_{1}-1\right) g=P_{1},\left(f-P_{1}\right)\left(g+P_{1}-1\right)=P_{1}\left(1-P_{1}\right)$, $f=P_{2} g, f+\left(P_{2}-1\right) g=P_{2}$ and $\left(f-P_{2}\right)\left(g+P_{2}-1\right)=P_{2}\left(1-P_{2}\right)$ holds, then in the same manner as in the proof of Lemma 2.8 we get a contradiction. Thus from Lemma 2.9 we get

$$
N_{(3}\left(r, \frac{1}{f-P_{1}}\right)+N_{(3}\left(r, \frac{1}{g-P_{1}}\right)+N_{(3}\left(r, \frac{1}{f-P_{2}}\right)+N_{(3}\left(r, \frac{1}{g-P_{2}}\right)=S(r, f) .
$$

From (3.2), (3.3) and (3.4) we get

$$
T(r, f)=N_{2)}\left(r, \frac{1}{f-P_{1}}\right)+S(r, f)
$$

and

$$
T(r, g)=N_{2)}\left(r, \frac{1}{g-P_{2}}\right)+S(r, f) .
$$

we discuss the following two cases.

Case 1. Suppose that

$$
\bar{N}_{(1,2)}\left(r, P_{1}, P_{2}\right)+\bar{N}_{(2,1)}\left(r, P_{1}, P_{2}\right)=S(r, f),
$$

where and in what follows, $\bar{N}_{(l, k)}\left(r, P_{1}, P_{2}\right)$ denotes the reduced counting function of those common zeros of $f-P_{1}$ and $g-P_{2}$, and each such common zero of $f-P_{1}$ and $g-P_{2}$ is of $f-P_{1}$ with multiplicity $l$, and of $g-P_{2}$ with multiplicity $k$. Then from (3.7) and the condition that $f-P_{1}$ and $g-P_{2}$ share 0 IM we see that $f-P_{1}$ and $g-P_{2}$ share $0 \mathrm{CM}^{*}$. This together with Lemma 2.10 and the condition that $f$ and $g$ share $0,1, \infty$ CM implies that $f$ is a quasi-Möbius transformation of $g$. By Lemma 2.11, we discuss the following two subcases.

Subcase 1.1. Suppose that $f$ and $g$ satisfy one of the three relations (i), (ii) and (vi) of Lemma 2.11. If $f$ and $g$ satisfy (i) of Lemma 2.11, from (3.5), (3.6) and the fact that $f-P_{1}$ and $g-P_{2}$ share $0 \mathrm{CM}^{*}$ we get $P_{1} P_{2}=1$, 
which is impossible. Similarly, if $f$ and $g$ satisfy (ii) of Lemma 2.11 we get $\left(P_{1}-1\right)\left(P_{2}-1\right)=1$, which is impossible.

If $f$ and $g$ satisfy (vi) of Lemma 2.11, in the same manner as above we get

$$
\left\{(c-1) P_{1}+1\right\} \cdot\left\{(c-1) P_{2}-c\right\}=-c .
$$

From (3.6) we get

$$
c=\frac{P_{2}\left(P_{1}-1\right)}{P_{1}\left(P_{2}-1\right)} .
$$

By substituting (2.16) and (2.17) into (vi) of Lemma 2.11 we get

$$
(1-c) e^{\beta}-e^{\alpha+\beta}+c e^{\alpha}+e^{2 \beta}-e^{2 \beta-\alpha}+c e^{\beta-\alpha}=c .
$$

If one of $e^{\alpha}, e^{\beta}$ and $e^{\beta-\alpha}$ is a constant, then $f$ is a Möbius transformation of $g$ such that $f$ and $g$ satisfy one of the six relations (i)-(vi) of Lemma 2.6. From the above supposition and in the same manner as above we get (3.9) and $c$ is a nonzero constant. Thus $P_{1}$ and $P_{2}$ share $0,1 \mathrm{CM}$. This together with Lemma 2.12 gives $P_{1}=P_{2}$, which is impossible. Thus none of $e^{\alpha}, e^{\beta}$ and $e^{\beta-\alpha}$ is a constant. If $e^{\alpha+\beta}$ is a constant, then $e^{2 \beta-\alpha}$ is not a constant. This together with (3.8), (3.10) and Lemma 2.7 gives $e^{\alpha+\beta}=-\left\{P_{2}\left(P_{1}-1\right)\right\} /\left\{P_{1}\left(P_{2}-1\right)\right\}$, which implies that $e^{\alpha+\beta}$ is a nonzero complex number. Thus $P_{1}$ and $P_{2}$ share 0, 1 CM. This together with Lemma 2.12 implies that $P_{1}=P_{2}$, which is impossible. If $e^{2 \beta-\alpha}$ is a constant, then $e^{\alpha+\beta}$ is not a constant. Proceeding as above, we get $e^{2 \beta-\alpha}=-\left\{P_{2}\left(P_{1}-1\right)\right\} /\left\{P_{1}\left(P_{2}-1\right)\right\}$, and so we get $P_{1}=P_{2}$, which is impossible.

Subcase 1.2. Suppose that $f$ and $g$ satisfy one of the three relations (iii), (iv) and (v) of Lemma 2.11. Combining (3.5), (3.6) and the fact that $f-P_{1}$ and $g-P_{2}$ share $0 \mathrm{CM}^{*}$, we get (i)-(iii) of Theorem 1.1 .

Case 2. Suppose that

$$
\bar{N}_{(1,2)}\left(r, P_{1}, P_{2}\right)+\bar{N}_{(2,1)}\left(r, P_{1}, P_{2}\right) \neq S(r, f) .
$$

From (3.11) we see that at least one of the two inequalities $\bar{N}_{(2,1)}\left(r, P_{1}, P_{2}\right) \neq$ $S(r, f)$ and $\bar{N}_{(1,2)}\left(r, P_{1}, P_{2}\right) \neq S(r, f)$, say

$$
\bar{N}_{(2,1)}\left(r, P_{1}, P_{2}\right) \neq S(r, f)
$$

holds. From (3.11) and (3.12) we will prove

$$
\bar{N}_{(1,2)}\left(r, P_{1}, P_{2}\right)=\bar{N}_{(2,1)}\left(r, P_{1}, P_{2}\right)+S(r, f) .
$$

In fact, from (2.16) and (2.17) we get

$$
\begin{gathered}
f-P_{1}=\frac{e^{\alpha}-P_{1} e^{\beta}+P_{1}-1}{e^{\beta}-1}, \\
g-P_{2}=\frac{e^{-\alpha}-P_{2} e^{-\beta}+P_{2}-1}{e^{-\beta}-1}
\end{gathered}
$$


and

$$
T(r, g)+T\left(r, e^{\alpha}\right)+T\left(r, e^{\beta}\right)=O(T(r, f))(r \notin E) .
$$

From (3.16) and Lemma 2.13 we get

$$
T\left(r, \alpha_{2}\right)+T\left(r, \beta_{2}\right)=S(r, f),
$$

where and in what follows,

$$
\alpha_{2}=\alpha^{\prime} \text { and } \beta_{2}=\beta^{\prime} .
$$

If $P_{1} \beta_{2}-P_{1} \alpha_{2}+P_{1}^{\prime}=0$, from (3.18) we deduce that there exists a nonzero complex number $A_{6}$ such that $e^{\alpha-\beta}=A_{6} P_{1}$, which is impossible. Thus $P_{1} \beta_{2}-$ $P_{1} \alpha_{2}+P_{1}^{\prime} \not \equiv 0$. Similarly, we get $\left(P_{1}-P_{1}^{2}\right) \beta_{2}+P_{1}^{\prime} \not \equiv 0, P_{1} \beta_{2}-P_{1} \alpha_{2}+P_{1}^{\prime} \not \equiv 0$ and $P_{1}^{\prime}+\left(1-P_{1}\right) \alpha_{2} \not \equiv 0$. Let $z_{0}$ be a zero of $f-P_{1}$ with multiplicity 2 , and of $g-P_{2}$ with multiplicity 1 , such that $z_{0} \notin S_{1} \cup S_{2} \cup S_{3} \cup S_{4}$, where

$$
\begin{gathered}
S_{1}=\left\{z: P_{1}(z) \beta_{2}(z)-P_{1}(z) \alpha_{2}(z)+P_{1}^{\prime}(z)=0\right\}, \\
S_{2}=\left\{z:\left\{P_{1}(z)-P_{1}^{2}(z)\right\} \beta_{2}(z)+P_{1}^{\prime}(z)=0\right\}, \\
S_{3}=\left\{z: P_{1}(z) \beta_{2}(z)-P_{1}(z) \alpha_{2}(z)+P_{1}^{\prime}(z)=0\right\}
\end{gathered}
$$

and

$$
S_{4}=\left\{z: P_{1}^{\prime}(z)+\left\{1-P_{1}(z)\right\} \alpha_{2}(z)=0\right\} .
$$

From (3.14) we get

$$
e^{\alpha\left(z_{0}\right)}-P_{1}\left(z_{0}\right) e^{\beta\left(z_{0}\right)}+P_{1}\left(z_{0}\right)-1=0
$$

and

$$
\alpha_{2}\left(z_{0}\right) e^{\alpha\left(z_{0}\right)}-e^{\beta\left(z_{0}\right)}\left\{P_{1}^{\prime}\left(z_{0}\right)+P_{1}\left(z_{0}\right) \cdot \beta_{2}\left(z_{0}\right)\right\}+P_{1}^{\prime}\left(z_{0}\right)=0 .
$$

From (3.23) and (3.24) we get

$$
e^{\alpha\left(z_{0}\right)}=\frac{\left\{P_{1}\left(z_{0}\right)-P_{1}^{2}\left(z_{0}\right)\right\} \beta_{2}\left(z_{0}\right)+P_{1}^{\prime}\left(z_{0}\right)}{P_{1}\left(z_{0}\right) \beta_{2}\left(z_{0}\right)-P_{1}\left(z_{0}\right) \alpha_{2}\left(z_{0}\right)+P_{1}^{\prime}\left(z_{0}\right)}
$$

and

$$
e^{\beta\left(z_{0}\right)}=\frac{P_{1}^{\prime}\left(z_{0}\right)+\left\{1-P_{1}\left(z_{0}\right)\right\} \alpha_{2}\left(z_{0}\right)}{P_{1}\left(z_{0}\right) \beta_{2}\left(z_{0}\right)-P_{1}\left(z_{0}\right) \alpha_{2}\left(z_{0}\right)+P_{1}^{\prime}\left(z_{0}\right)} .
$$

Let

$$
f_{1}=\frac{\left(P_{1} \beta_{2}-P_{1} \alpha_{2}+P_{1}^{\prime}\right) e^{\alpha}}{\left(P_{1}-P_{1}^{2}\right) \beta_{2}+P_{1}^{\prime}}, \quad f_{2}=\frac{\left(P_{1} \beta_{2}-P_{1} \alpha_{2}+P_{1}^{\prime}\right) e^{\beta}}{P_{1}^{\prime}+\left(1-P_{1}\right) \alpha_{2}}
$$

and

$$
T(r)=T\left(r, f_{1}\right)+T\left(r, f_{2}\right), \quad S(r)=o(T(r))(r \rightarrow \infty, r \notin E) .
$$

From (3.16), (3.17), (3.27) and (3.28) we get

$$
S(r)=S(r, f)
$$


and

$$
\bar{N}\left(r, f_{j}\right)+\bar{N}\left(r, \frac{1}{f_{j}}\right)=S(r)(j=1,2) .
$$

From $(3.25)-(3.27)$ we get $f_{1}\left(z_{0}\right)=f_{2}\left(z_{0}\right)=1$, and so

$$
\bar{N}_{(2,1)}\left(r, P_{1}, P_{2}\right) \leq N_{0}\left(r, 1 ; f_{1}, f_{2}\right)+S(r) .
$$

From (3.12), (3.29) and (3.31) we get

$$
N_{0}\left(r, 1 ; f_{1}, f_{2}\right) \neq S(r) .
$$

From (3.27), (3.29), (3.30), (3.32) and Lemma 2.14 we know that there exist two integers $s$ and $t(|s|+|t|>0)$ such that

$$
f_{1}^{s} \cdot f_{2}^{t}=1 \text {. }
$$

From (2.14), (2.15), (3.16), (3.17), (3.27), (3.33) and Lemma 2.15 we get

$$
T(r, f)=T(r, g)+S(r, f) .
$$

Again from (2.14) and (2.15) we get

$$
\frac{e^{\beta\left(z_{0}\right)}}{e^{\alpha\left(z_{0}\right)}}=\frac{P_{2}\left(z_{0}\right)}{P_{1}\left(z_{0}\right)}, \quad \frac{1}{e^{\alpha\left(z_{0}\right)}}=\frac{P_{2}\left(z_{0}\right)-1}{P_{1}\left(z_{0}\right)-1} .
$$

Since (3.24) can be rewritten as

$$
\alpha_{2}\left(z_{0}\right)-\frac{e^{\beta\left(z_{0}\right)}}{e^{\alpha\left(z_{0}\right)}}\left\{P_{1}^{\prime}\left(z_{0}\right)+P_{1}\left(z_{0}\right) \cdot \beta_{2}\left(z_{0}\right)\right\}+\frac{P_{1}^{\prime}\left(z_{0}\right)}{e^{\alpha\left(z_{0}\right)}}=0 .
$$

From (3.35) and (3.36) we get

(3.37) $\alpha_{2}\left(z_{0}\right)-\frac{P_{2}\left(z_{0}\right)}{P_{1}\left(z_{0}\right)} \cdot\left\{P_{1}^{\prime}\left(z_{0}\right)+P_{1}\left(z_{0}\right) \cdot \beta_{2}\left(z_{0}\right)\right\}+P_{1}^{\prime}\left(z_{0}\right) \cdot \frac{P_{2}\left(z_{0}\right)-1}{P_{1}\left(z_{0}\right)-1}=0$.

From (3.12) and (3.37) we get

$$
\alpha_{2}-\frac{P_{2}\left(P_{1}^{\prime}+P_{1} \cdot \beta_{2}\right)}{P_{1}}+\frac{\left(P_{2}-1\right) P_{1}^{\prime}}{P_{1}-1}=0 .
$$

From $(3.5),(3.6),(3.34)$ and the condition that $f-P_{1}$ and $g-P_{2}$ share 0 IM we get

$$
\begin{aligned}
T(r, f)-T(r, g) & =N_{2)}\left(r, \frac{1}{f-P_{1}}\right)-N_{2)}\left(r, \frac{1}{g-P_{2}}\right)+S(r, f) \\
& =\bar{N}_{(2,1)}\left(r, P_{1}, P_{2}\right)-\bar{N}_{(1,2)}\left(r, P_{1}, P_{2}\right)=S(r, f),
\end{aligned}
$$

namely

$$
\bar{N}_{(2,1)}\left(r, P_{1}, P_{2}\right)-\bar{N}_{(1,2)}\left(r, P_{1}, P_{2}\right)=S(r, f),
$$

which together with (3.12) implies

$$
\bar{N}_{(2,1)}\left(r, P_{1}, P_{2}\right) \neq S(r, f) \quad \text { and } \quad \bar{N}_{(1,2)}\left(r, P_{1}, P_{2}\right) \neq S(r, f) .
$$


From (3.15), the right inequality of (3.39) and in the same manner as in the proof of (3.38) we get

$$
-\alpha_{2}-\frac{P_{1}\left(P_{2}^{\prime}-P_{2} \beta_{2}\right)}{P_{2}}+\frac{\left(P_{1}-1\right) P_{2}^{\prime}}{P_{2}-1}=0 .
$$

By rewriting (3.38) and (3.40) we get

$$
\alpha_{2}-P_{2} \beta_{2}=\frac{\left(1-P_{2}\right) P_{1}^{\prime}}{P_{1}-1}+\frac{P_{2} P_{1}^{\prime}}{P_{1}}
$$

and

$$
-\alpha_{2}+P_{1} \beta_{2}=\frac{\left(1-P_{1}\right) P_{2}^{\prime}}{P_{2}-1}+\frac{P_{1} P_{2}^{\prime}}{P_{2}}
$$

respectively. From (3.18) we see that $\alpha_{2}$ and $\beta_{2}$ are entire functions. Thus from (3.41) and (3.42) we see that $P_{1}$ and $P_{2}$ share 0 and 1 IM. This together with Lemma 2.12 implies $P_{1}=P_{2}$, which is impossible. Theorem 1.1 is thus completely proved.

Proof of Theorem 1.2. Proceeding as in the beginning of the proof of Theorem 1.1 we see that if $f$ and $g$ are polynomials, then the conclusion of Theorem 1.2 holds. Next we suppose that $f$ and $g$ are two distinct transcendental entire functions. Then we have (2.14)-(2.17). Proceeding as in the proof of Theorem 1.1 we have (3.5) and (3.6). From Lemma 2.1 and Lemma 2.16 we get

$$
T(r, f)=T(r, g)+S(r, f) .
$$

From $(3.5),(3.6),(3.43)$ and the condition $f-P_{1}=0 \Longrightarrow g-P_{2}=0$ we deduce that $f-P_{1}$ and $g-P_{2}$ share $0 \mathrm{IM}^{*}$. Next in the same manner as in Case 1 and Case 2 in the proof of Theorem 1.1 we get (i)-(iii) of Theorem 1.1.

If $f$ and $g$ satisfy (i) of Theorem 1.1, then 0 is a Picard exceptional value of $f$ and $g$. Thus $f=e^{\alpha_{3}}$ and $g=e^{\beta_{3}}$, where $\alpha_{3}$ and $\beta_{3}$ are entire functions. Thus (i) of Theorem 1.1 can be rewritten as $e^{\alpha_{3}}+e^{\beta_{3}}=1$. From this and Lemma 2.7 we get a contradiction.

If $f$ and $g$ satisfy (ii) of Theorem 1.1, then

$$
\bar{N}\left(r, \frac{1}{f-1}\right)+\bar{N}\left(r, \frac{1}{f-P_{1} / P_{2}}\right)=S(r, f) .
$$

From (3.44) and Nevanlinna's three small functions theorem we get

$$
T(r, f) \leq \bar{N}\left(r, \frac{1}{f-1}\right)+\bar{N}\left(r, \frac{1}{f-P_{1} / P_{2}}\right)=S(r, f),
$$

which is impossible.

If $f$ and $g$ satisfy (iii) of Theorem 1.1, then $N(r, 1 / f)=O(\log r)$. Thus

$$
f=P_{3} e^{\alpha_{4}} \quad \text { and } \quad g=P_{4} e^{\beta_{4}},
$$


where $P_{3}$ and $P_{4}$ are nonzero polynomials, $\alpha_{4}$ and $\beta_{4}$ are nonconstant entire functions. By substituting (3.45) into (iii) of Theorem 1.1 we get

$$
P_{3} e^{\alpha_{4}}-\frac{P_{4}\left(P_{1}-1\right)}{P_{2}-1} \cdot e^{\beta_{4}}=\frac{P_{2}-P_{1}}{P_{2}-1} .
$$

From (3.46) and Lemma 2.7 we get a contradiction. Theorem 1.2 is thus completely proved.

Acknowledgement. The authors wish to express their thanks to the referee for his valuable suggestions and comments.

\section{References}

[1] A. H. H. Al-Khaladi, Unicity theorems for meromorphic functions, Kodai Math. J. 23 (2000), no. $1,27-34$.

[2] T. C. Alzahary, Weighted sharing three values and Brosch's theorem, J. Math. Anal. Appl. 323 (2006), no. 1, 8-25.

[3] T. C. Alzahary and H. X. Yi, Weighted sharing three values and uniqueness of meromorphic functions, J. Math. Anal. Appl. 295 (2004), no. 1, 247-257.

[4] _ Meromorphic functions that weighted sharing three values and one pair, Kodai Math. J. 29 (2006), no. 1, 13-30.

[5] G. Brosch, Eindeutigkeitssätze für meromorphe Funktionen, Thesis, Technical University of Aachen, 1989.

[6] F. Gross, On the distribution of values of meromorphic functions, Trans. Amer. Math. Soc. 131 (1968), 199-214.

[7] G. G. Gundersen, Meromorphic functions that share three or four values, J. London Math. Soc. (2) 20 (1979), no. 3, 457-466.

[8] W. K. Hayman, Meromorphic Functions, The Clarendon Press, Oxford, 1964.

[9] I. Lahiri, Weighted sharing and uniqueness of meromorphic functions, Nagoya Math. J. 161 (2001), 193-206.

[10] Weighted sharing of three values and uniqueness of meromorphic functions, Kodai Math. J. 24 (2001), no. 3, 421-435.

[11] I. Lahiri and P. Sahoo, On a result of G. Brosch, J. Math. Anal. Appl. 331 (2007), no. $1,532-546$.

[12] X. M. Li and H. X. Yi, The uniqueness theorems of meromorphic functions sharing three values and one pair of values, J. Math. Anal. Appl. 339 (2008), no. 1, 609-621.

[13] P. Li and C. C. Yang, On two meromorphic functions that share pairs of small functions, Complex Variables Theory Appl. 32 (1997), no. 2, 177-190.

[14] X. M. Li and H. X. Yi, Weighted sharing of meromorphic functions relative to small functions, J. Math. Anal. Appl. 343 (2008), no. 2, 919-931.

[15] _ Uniqueness of meromorphic functions whose derivatives share four small functions, J. Math. Anal. Appl. 352 (2009), no. 2, 573-582.

[16] A. Z. Mokhon'ko, On the Nevanlinna characteristics of some meromorphic functions, in: Theory of Functions, Functional Analysis and Their Applications, vol. 14, Izd-vo Khar'kovsk. Un-ta, 1971, pp. 83-87.

[17] R. Nevanlinna, Einige Eindeutigkeitssätze in der Theorie der Meromorphen Funktionen, Acta Math. 48 (1926), no. 3-4, 367-391.

[18] C. C. Yang and H. X. Yi, Uniqueness Theory of Meromorphic Functions, Mathematics and its Applications, 557. Kluwer Academic Publishers Group, Dordrecht, 2003.

[19] H. X. Yi, Meromorphic functions with weighted sharing of three values, Complex Var. Theory Appl. 50 (2005), no. 12, 923-934.

[20] - On a result of Gross and Yang, Tohoku Math. J. (2) 42 (1990), no. 3, 419-428. 
[21] H. X. Yi and Y. H. Li, On Osgood-Yang's conjecture and Mues' conjecture, Nagoya Math. J. 170 (2003), 163-173.

[22] Q. C. Zhang, Meromorphic functions sharing three values, Indian J. Pure Appl. Math. 30 (1999), no. 7, 667-682.

\section{XIAO-Min Li}

Department of Mathematics

OCEAN University of ChinA

Qingdao, Shandong 266100, P. R. China

AND

Department of Physics and Mathematics

University of EAstern Finland

P. O. Box 111, 80101 Joensuu, Finland

E-mail address: xmli01267@gmail.com

HONG-XUN YI

Department of Mathematics

SHANDONG UNIVERSITY

Jinan, Shandong 250100, P. R. China

E-mail address: hxyi@sdu.edu.cn 